\title{
Src/caveolin-1-regulated EGFR activation antagonizes TRAIL-induced apoptosis in gastric cancer cells
}

\author{
LING XU ${ }^{1}$, XIUJUAN QU ${ }^{1}$, HEMING LI ${ }^{1}, \mathrm{CE} \mathrm{LI}^{1}$, JING LIU ${ }^{1}$, HUACHUAN ZHENG $^{2}$ and YUNPENG LIU ${ }^{1}$ \\ ${ }^{1}$ Department of Medical Oncology, The First Hospital of China Medical University, Heping, Shenyang 110001; \\ ${ }^{2}$ Cancer Research Center, The First Affiliated Hospital of Liaoning Medical University, Jinzhou, Liaoning 121001, P.R. China
}

Received March 6, 2014; Accepted April 17, 2014

DOI: $10.3892 /$ or.2014.3183

\begin{abstract}
Gastric cancer cells are insensitive to tumor necrosis factor-related apoptosis-inducing ligand (TRAIL), and we recently showed that lipid raft-regulated epidermal growth factor receptor (EGFR) activation antagonized TRAILinduced apoptosis. However, it is not clear whether caveolin-1, an essential structural constituent of lipid rafts, regulates lipid raft-mediated EGFR activation. We report here that TRAIL induced the translocation of EGFR into lipid rafts and its activation in gastric cancer SGC-7901 and MGC-803 cells. Simultaneously, caveolin-1 was also activated. Knockdown of caveolin-1 partially prevented EGFR activation and increased TRAIL sensitivity. Moreover, TRAIL promoted the translocation of Src into lipid rafts and its activation, as well as the interaction of Src with both EGFR and caveolin-1. A Src inhibitor prevented these interactions and the activation of caveolin-1 and EGFR, and thus enhanced TRAIL-induced apoptosis. These data suggest that Src activates EGFR through the interaction of both Src-EGFR and Src-caveolin-1, and then antagonizes TRAIL-induced apoptosis in gastric cancer cells.
\end{abstract}

\section{Introduction}

Gastric cancer is highly prevalent in developing nations, particularly in China, yet there is a lack of effective drugs for the treatment of advanced gastric cancer (1). Recently, tumor necrosis factor-related apoptosis-inducing ligand (TRAIL) has received much attention due to its high specificity and low toxicity in anticancer treatment $(2,3)$. However, various types

Correspondence to: Professor Yunpeng Liu or Professor Xiujuan Qu, Department of Medical Oncology, The First Hospital of China Medical University, No. 155 North Nanjing Street, Heping, Shenyang 110001, P.R. China

E-mail: cmuliuyunpeng@hotmail.com

E-mail: qu_xiujuan@hotmail.com

Abbreviations: EGFR, epidermal growth factor receptor; PARP, poly(ADP-ribose) polymerase; TGF, transforming growth factor; TRAIL, TNF-related apoptosis-inducing ligand; VEGF, vascular endothelial growth factor

Key words: TRAIL, caveolin-1, Src, EGFR of gastric cancer cells are resistant to TRAIL $(4,5)$ and the mechanism of TRAIL resistance is not fully understood.

Survival pathway activation is known to be involved in TRAIL resistance. For example, a previous study showed that TRAIL-induced activation of epidermal growth factor receptor (EGFR) resulted in TRAIL resistance in colorectal cancer cells (6), and we recently demonstrated that TRAIL induced EGFR activation in TRAIL-resistant gastric cancer cells. The plasma membrane lipid rafts regulate TRAIL-induced EGFR activation (7). Lipid rafts are composed of multiple molecules, but it is not clear which molecule plays the most important role in the regulation of EGFR activation. The scaffolding protein caveolin-1 is enriched in lipid rafts, where it takes on a specialized role in regulating the activity of cell signaling receptors and their downstream effectors, such as EGFR (8). However, although caveolin-1 phosphorylation was shown to be required for stretch-induced EGFR/Akt activation in mesangial cells (9), its involvement in the regulation of TRAIL-induced EGFR activation warrants further investigation.

EGFR is typically activated by its ligands, but it can also be regulated by the non-receptor tyrosine kinase Src. In non-small cell lung cancer cells, transfection with Src short hairpin RNA diminished phosphorylation of EGFR and ErbB2 and increased sensitivity to EGFR tyrosine kinase inhibitor (10). In mesangial cells, Src also promoted the phosphorylation of caveolin-1 and vascular endothelial growth factor (VEGF)-induced fibronectin upregulation (11). Moreover, Src activation increased caveolin-1 phosphorylation and promoted nitric oxide synthase/caveolin-1 binding in vascular endothelial cells (12). However, the mechanism by which Src and caveolin-1 regulate TRAIL-induced EGFR activation is unknown.

In the present study, we showed that Src interacted with both EGFR and caveolin-1 to regulate EGFR activation, thus antagonizing TRAIL-induced apoptosis in gastric cancer cells.

\section{Materials and methods}

Reagents and antibodies. Recombinant human TRAIL was purchased from Cytolab/Peprotech Asia (USA). Src inhibitor PP2 was obtained from Sigma (St. Louis, MO, USA). Anti-EGFR, anti-phospho-EGFR (Tyr1068), anti-Src, anti-phospho-Src (Tyr416), anti-Akt and anti-phospho-Akt (Ser473) antibodies were obtained from Cell Signaling 
Technology (USA). Anti-ERK, anti-p-ERK 1/2 (Thr 202/ Tyr 204), and anti-actin antibodies were obtained from Santa Cruz Biotechnology (USA). Anti-caveolin-1 and antiphospho-caveolin-1 (Tyr14) antibodies were obtained from BD Technology Co. (USA).

Cell cultures. SGC-7901 and MGC-803 cells were obtained from the Type Culture Collection of the Chinese Academy of Sciences (China). The cells were cultured in RPMI-1640 medium containing $10 \%$ heat-inactivated fetal bovine serum at $37^{\circ} \mathrm{C}$ under an atmosphere of $95 \%$ air and $5 \% \mathrm{CO}_{2}$.

Flow cytometric analysis. SGC-7901 and MGC-803 cells were incubated with the indicated concentrations of agents for the indicated times. After being fixed with ice-cold $70 \%$ ethanol overnight, the samples were incubated with $20 \mu \mathrm{g} / \mathrm{ml}$ RNase A and $10 \mu \mathrm{g} / \mathrm{ml}$ propidium iodide for $30 \mathrm{~min}$ in the dark. Finally, the samples were evaluated by flow cytometry, and the data were analyzed with CellQuest software (Becton-Dickinson, San Jose, CA, USA).

Small interfering RNA transfections. Cavolin-1 small interfering RNA (siRNA) was obtained from Shanghai GeneChem Co. Ltd. (China). Cavolin-1 siRNA was synthesized: 5'-AACC AGAAGGGACACACAGUU-3' (sense) and 5'-AACUGUGUG UCCCUUCUGGUU-3' (antisense). SGC-7901 and MGC-803 cells were transiently transfected with cavolin-1 siRNA using Lipofectamine 2000 reagent (Invitrogen, USA), according to the manufacturer's instructions.

Isolation of lipid rafts. SGC-7901 and MGC-803 cells were solubilized in $150 \mu \mathrm{l}$ of prechilled TXNE buffer $(50 \mathrm{mM}$ Tris-HCl pH 7.4, $150 \mathrm{mM} \mathrm{NaCl}, 5 \mathrm{mM}$ EDTA and $0.1 \%$ Triton X-100) containing protease inhibitors (chymostatin, leupeptin, antipain and pepstatin). Subsequently, the cells were moved into 35\% OptiPrep (Axis-Shield, Norway) in polyallomer ultra tubes (Sorvall Instruments, USA) by adding $210 \mu \mathrm{l}$ of $60 \%$ OptiPrep/0.1\% Triton X-100. The cell lysates were covered with $3.5 \mathrm{ml} 30 \%$ OptiPrep in TXNE buffer and $300 \mu 1$ TXNE buffer. After spinning in the ultracentrifuge (4 h, 200,000 x g, $4^{\circ} \mathrm{C}$ ) (Sorvall/Kendro, USA), six fractions were collected from the top. The proteins in fractions 1-2 were taken as the lipid raft fractions.

Immunoprecipitation and western blot analysis. SGC-7901 and MGC-803 cells were solubilized in 1\% Triton lysis buffer (1\% Triton X-100, $50 \mathrm{mM}$ Tris-Cl pH 7.4, $150 \mathrm{mM} \mathrm{NaCl}$, $10 \mathrm{mM}$ EDTA, $100 \mathrm{mM} \mathrm{NaF}, 1 \mathrm{mM} \mathrm{Na}_{3} \mathrm{VO}_{4}, 1 \mathrm{mM}$ PMSF, $2 \mu \mathrm{g} / \mathrm{ml}$ aprotinin). For immunoprecipitation, cell lysates were mixed with Src or caveolin-1 antibody and $20 \mathrm{ml}$ of protein G-sepharose beads (Cell Signaling Technology) at $4^{\circ} \mathrm{C}$ overnight. The immunoprecipitated proteins were eluted by heat treatment at $100^{\circ} \mathrm{C}$ for 5 min with $2 \mathrm{X}$ sampling buffer. For the preparation of total cell lysates, cell lysate proteins were separated by sodium dodecyl sulfate-polyacrylamide gel electrophoresis and electrophoretically transferred to a nitrocellulose membrane (Immoblin-P; Millipore, Bedford, MA, USA). The membranes were blocked with 5\% skim milk in TBST buffer (10 mM Tris-Cl pH 7.4, $150 \mathrm{mM} \mathrm{NaCl}, 0.1 \%$ Tween-20) at room temperature and incubated overnight at $4^{\circ} \mathrm{C}$ with the indicated primary antibodies. After the appropriate secondary antibodies were added at room temperature, the proteins were detected with enhanced chemiluminescence reagent (SuperSignal Western Pico Chemiluminescent Substrate; Pierce, USA) and visualized with the Electrophoresis Gel Imaging Analysis System (DNR Bio-Imaging Systems, Israel).

In situ proximity ligation assay. In situ proximity ligation assay (PLA) was carried out to detect the interactions of Src, EGFR or caveolin-1. We used Duolink in situ PLA (Olink Bioscience). In the assay, oligonucleotide-conjugated 'PLA probe' antibodies are directed against primary antibodies for Src, EGFR or caveolin-1. Annealing of the 'PLA probes' occurs when Src, EGFR or caveolin-1 are in close proximity, which initiates the amplification of repeat sequences recognized by the fluorescently labeled oligonucleotide probe. SGC-7901 and MGC-803 cells were seeded on 8-well chamber slides and stimulated as described above. Then cells were washed with ice-cold phosphate-buffered saline (PBS) and fixed with 4\% paraformaldehyde PBS for $30 \mathrm{~min}$ at room temperature. The cells were permeabilized with $0.2 \%$ Triton X-100 PBS for $10 \mathrm{~min}$ at room temperature. After that, the cells were assayed with Duolink in situ PLA. Anti-Src Ab and anti-EGFR Ab, or anti-Src Ab and anti-caveolin-1 Ab or anti-EGFR Ab and anticaveolin-1 Ab were used as primary antibodies. PLA probe anti-mouse Minus and PLA probe anti-rabbit Plus were used as 2 PLA probes. For detection, Duolink detection kit 563 was used. ProLong Gold Antifade Reagent with 4',6-diamidino2-phenylindole (DAPI; Molecular Probes) was used as the mounting medium. The specimens were observed using a fluorescence microscope (FV1000S-SIM/IX81; Olympus, Japan).

Statistical analysis. Data were confirmed in three independent experiments, and are expressed as the means \pm standard deviation (SD). Differences between groups were compared using the Student's t-test. SPSS 18.0 computer software was used for statistical analysis, and $\mathrm{P}<0.05$ was considered to indicate a statistically significant result.

\section{Results}

TRAIL induces EGFR and Src translocation into lipid rafts and activates EGFR, Src and caveolin-1 in gastric cancer cells. Since caveolin-1 is an essential structural constituent of lipid rafts in the cell membrane (13), we isolated membrane lipid rafts by ultracentrifugation and located them using caveolin-1 (fractions 1 and 2; Fig. 1A). TRAIL was used at $100 \mathrm{ng} / \mathrm{ml}$ in all experiments (14), and was shown to induce EGFR and Src translocation into caveolin-1-enriched lipid raft fractions (1 and 2) in SGC-7901 and MGC-803 cells (Fig. 1A). TRAIL also induced the activation of Src, caveolin-1, EGFR and downstream Akt/ERK in a temporal manner (Fig. 1B). These data suggest that Src and caveolin-1 are involved in EGFR activation in TRAIL-resistant gastric cancer cells.

Knockdown of caveolin-1 enhances TRAIL sensitivity via inhibition of EGFR activation in gastric cancer cells. To further delineate the role of caveolin-1 in TRAIL resistance, 

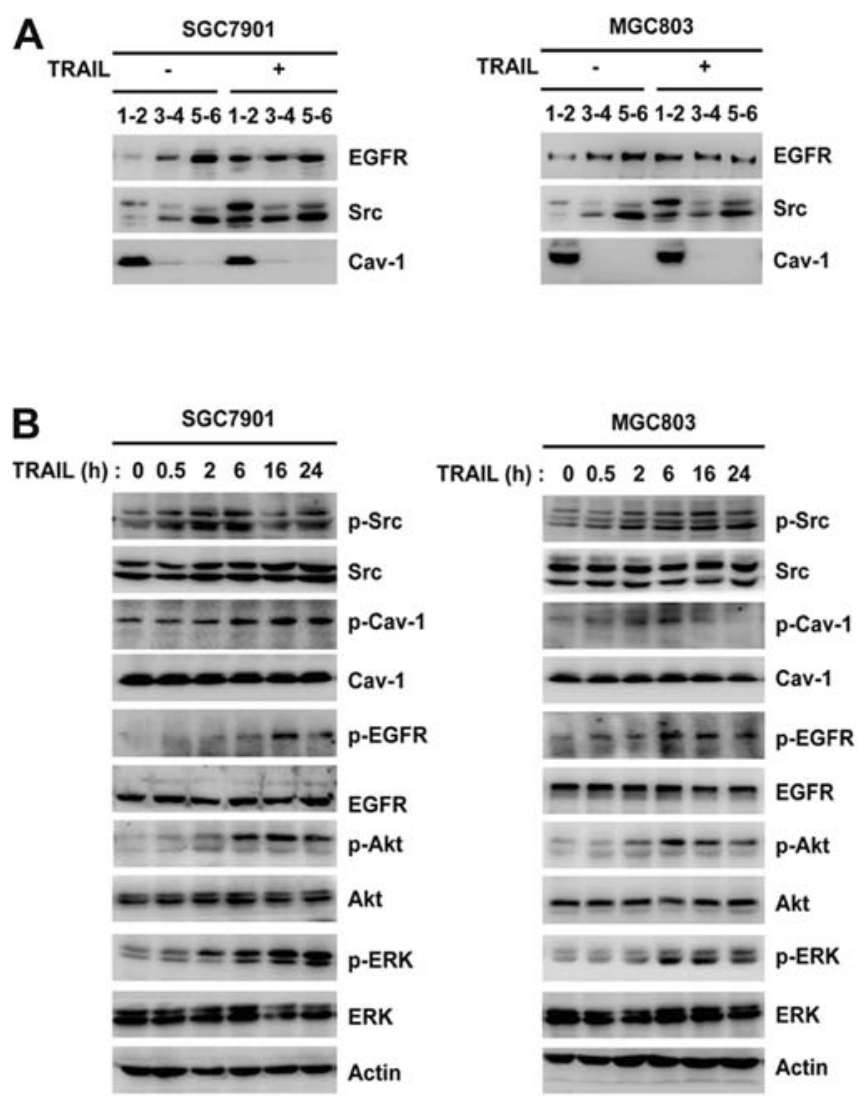

Figure 1. TRAIL induces the translocation of EGFR and Src into lipid rafts and the activation of EGFR, Src and caveolin-1 in gastric cancer cells. (A) SGC-7901 and MGC-803 cells were treated with $100 \mathrm{ng} / \mathrm{ml}$ TRAIL for $6 \mathrm{~h}$, and were then lysed and fractionated by ultracentrifuge. Locations of lipid rafts (fractions 1-2) were determined using caveolin-1. The distribution of EGFR and Src was analyzed by western blotting. (B) SGC-7901 and MGC-803 cells were incubated with $100 \mathrm{ng} / \mathrm{ml}$ TRAIL for $0.5,2,6$, 16 and $24 \mathrm{~h}$. The phosphorylation of Src, caveolin-1, EGFR, Akt and ERK was analyzed by western blotting. TRAIL, tumor necrosis factor-related apoptosis-inducing ligand; EGFR, epidermal growth factor receptor.

SGC-7901 and MGC-803 cells were transiently transfected with caveolin-1 siRNA for $48 \mathrm{~h}$ prior to TRAIL treatment for $6 \mathrm{~h}$. As shown in Fig. 2A, knockdown of caveolin-1 partially prevented EGFR and downstream Akt/ERK activation in the SGC-7901 and MGC-803 cells. Caveolin-1 siRNA had no significant effect on apoptosis (Fig. 2B). However, preincubation with caveolin-1 siRNA for $48 \mathrm{~h}$ followed by TRAIL treatment for $24 \mathrm{~h}$ resulted in a significant increase in apoptosis compared with TRAIL treatment alone in the SGC-7901 and MGC-803 cells $(12.70 \pm 3.24$ vs. $2.70 \pm 1.34 \%$ and $13.90 \pm 3.07$ vs. $4.40 \pm 1.57 \%$, respectively, $\mathrm{P}<0.05$; Fig. 2B). Caspase-3 and -8 activation and poly(ADP-ribose) polymerase (PARP) cleavage were also detected (Fig. 2C). These results suggest that caveolin-1 promotes EGFR activation and antagonizes TRAIL-induced apoptosis in gastric cancer cells.

Inhibition of Src activation increases TRAIL sensitivity by the inhibition of caveolin-1 and EGFR activation in gastric cancer cells. To investigate the effect of Src on caveolin-1-regulated EGFR activation, SGC-7901 and MGC-803 cells were pretreated with $10 \mu \mathrm{M}$ PP2 (a specific Src inhibitor) for $1 \mathrm{~h}$
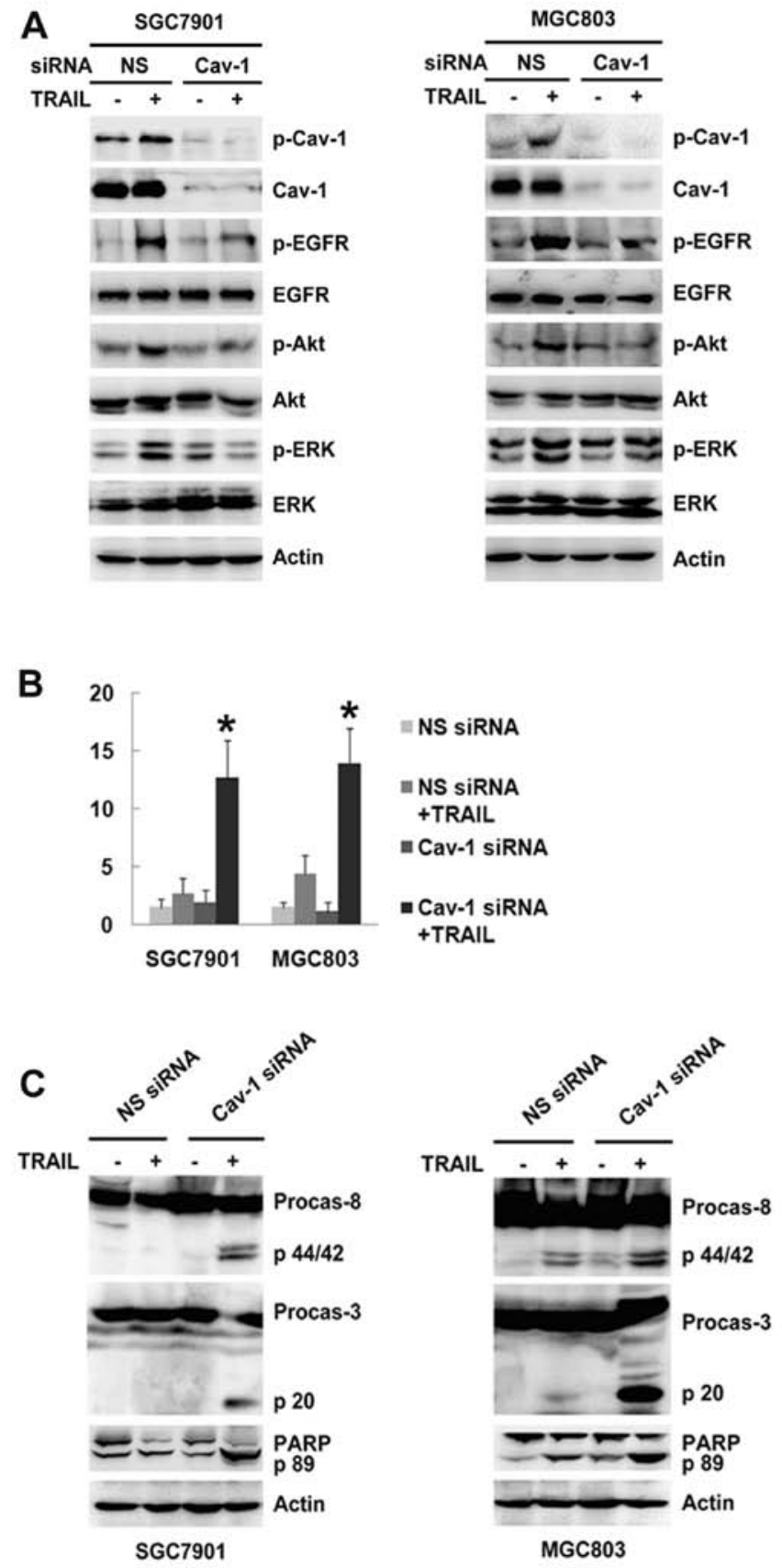

Figure 2. Knockdown of caveolin-1 enhances TRAIL sensitivity through the inhibition of EGFR activation in gastric cancer cells. (A) SGC-7901 and MGC-803 cells were transiently transfected with caveolin-1 siRNA for $48 \mathrm{~h}$, followed by $100 \mathrm{ng} / \mathrm{ml}$ TRAIL for $6 \mathrm{~h}$. The phosphorylation of caveolin-1, EGFR, Akt and ERK was analyzed by western blotting. (B) SGC-7901 and MGC-803 cells were transiently transfected with caveolin-1 siRNA for $48 \mathrm{~h}$, followed by $100 \mathrm{ng} / \mathrm{ml}$ TRAIL for $24 \mathrm{~h}$. Cell apoptosis was quantified by flow cytometry. "P<0.05, incubation with TRAIL in caveolin-1 siRNA vs. that in non-silenced siRNA (NS siRNA). (C) SGC-7901 and MGC-803 cells were treated under similar conditions to those in Fig. 2A. The expression of caspase- 3 and -8 and PARP was analyzed by western blotting. TRAIL, tumor necrosis factor-related apoptosis-inducing ligand; EGFR, epidermal growth factor receptor; siRNA, small interfering RNA; PARP, poly(ADP-ribose) polymerase.

before TRAIL treatment for $6 \mathrm{~h}$. As shown in Fig. 3A, this not only inhibited TRAIL-induced phosphorylation of caveolin-1, but also prevented EGFR and downstream Akt/ERK activation. However, knockdown of caveolin-1 did not influence Src 

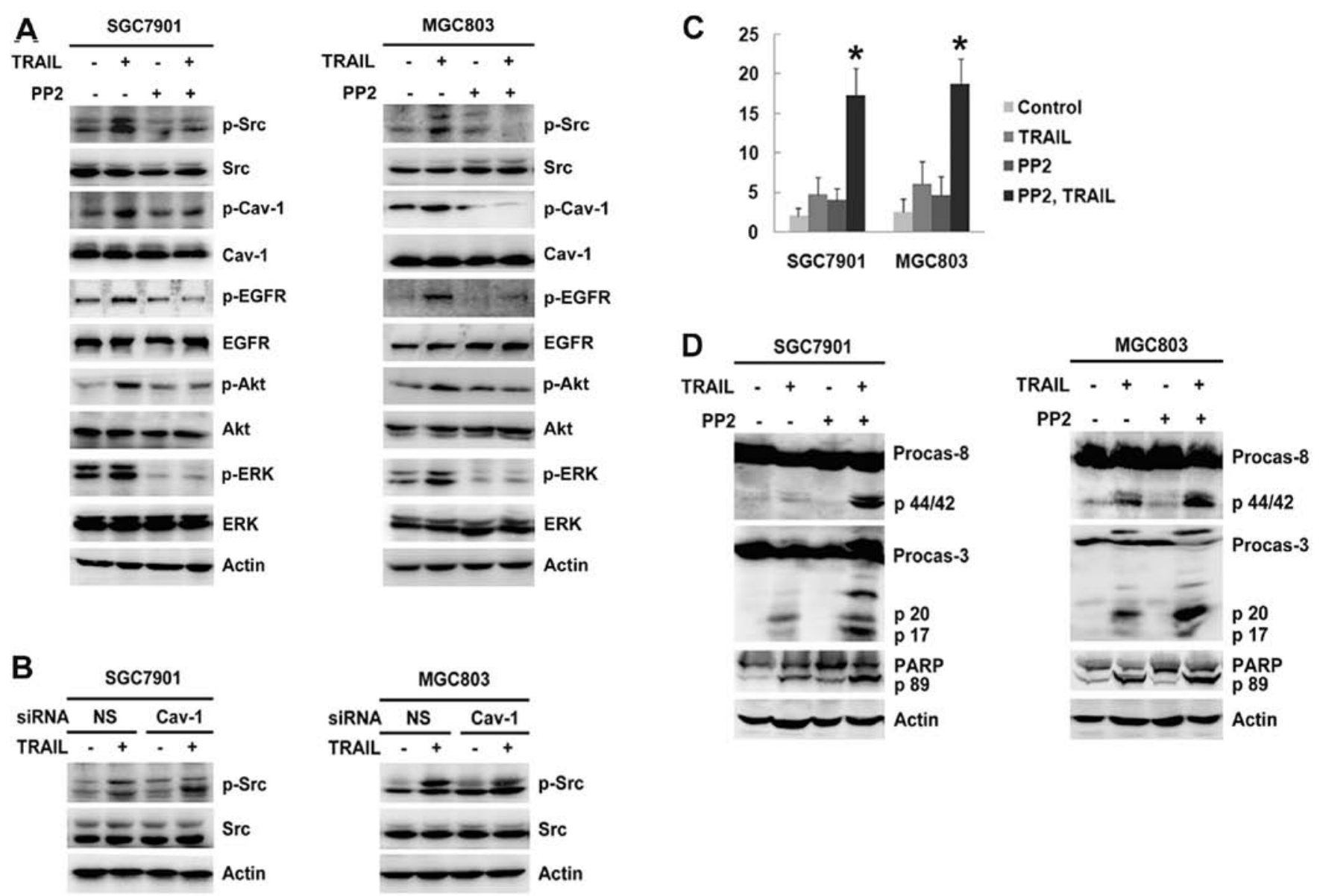

Figure 3. Inhibition of Src activation increases TRAIL sensitivity via the inhibition of caveolin-1 and EGFR activation in gastric cancer cells. (A) SGC-7901 and MGC-803 cells were preincubated with $10 \mu \mathrm{M}$ Src inhibitor PP2 for $1 \mathrm{~h}$, and then treated with $100 \mathrm{ng} / \mathrm{ml}$ TRAIL for $6 \mathrm{~h}$. The phosphorylation of Src, caveolin-1, EGFR, Akt and ERK was analyzed by western blotting. (B) SGC-7901 and MGC-803 cells were transiently transfected with caveolin-1 siRNA for $48 \mathrm{~h}$, followed by $100 \mathrm{ng} / \mathrm{ml}$ TRAIL for $6 \mathrm{~h}$. The phosphorylation of Src was analyzed by western blotting. (C) SGC-7901 and MGC-803 cells were incubated with $10 \mu \mathrm{M}$ PP2 for $1 \mathrm{~h}$, and then treated with $100 \mathrm{ng} / \mathrm{ml}$ TRAIL for $24 \mathrm{~h}$. Cell apoptosis was quantified using flow cytometry. ${ }^{*} \mathrm{P}<0.05$, incubation with PP2 and TRAIL vs. with TRAIL alone. (D) SGC-7901 and MGC-803 cells were treated under similar conditions to those in A. The expression of caspase-3, and -8, and PARP was analyzed by western blotting. TRAIL, tumor necrosis factor-related apoptosis-inducing ligand; EGFR, epidermal growth factor receptor; siRNA, small interfering RNA; PARP, poly(ADP-ribose) polymerase.

activation (Fig. 3B). PP2 alone had little effect on cell apoptosis after TRAIL treatment for $24 \mathrm{~h}$ (Fig. 3C). However, compared with TRAIL alone, preincubation with PP2 significantly increased the rate of cell apoptosis induced by TRAIL in the SGC-7901 and MGC- 803 cells $(17.32 \pm 3.43$ vs. $4.72 \pm 2.15 \%$ and $18.74 \pm 3.14$ vs. $6.10 \pm 2.83 \%$, respectively, $\mathrm{P}<0.05$; Fig. $3 \mathrm{C}$ ). Meanwhile, cleavage of caspase- 8 and -3 , and PARP was detected in cells treated with TRAIL and PP2 (Fig. 3D). These results suggest that Src promotes caveolin-1-regulated EGFR activation and antagonizes TRAIL-induced apoptosis in gastric cancer cells.

TRAIL induces the interaction of Src with both EGFR and caveolin-1 in gastric cancer cells. To identify the regulatory mechanism of Src and caveolin-1 in EGFR activation, we investigated the interaction of Src with EGFR and caveolin-1. The Src-EGFR and Src-caveolin-1 interactions were detected by immunoprecipitation (Fig. 4A). In the untreated SGC-7901 and MGC-803 cells, Src interacted weakly with both EGFR and caveolin-1 (Fig. 4A). However, the interaction of Src with both EGFR and caveolin-1 was strengthened after TRAIL treatment (Fig. 4A). The Src-EGFR and Src-caveolin-1 interactions were also detected by the proximity ligation assay Duolink (Fig. 4B). This technique confirmed the promotion of these interactions by TRAIL (Fig. 4B), although no interaction between EGFR and caveolin-1 was detected (data not shown). These data indicate that Src is an important mediator between EGFR and caveolin-1 in TRAIL-induced EGFR activation.

Inhibition of Src activation prevents the interaction of Src with both EGFR and caveolin-1 in gastric cancer cells. As shown in Fig. 5A, the Src inhibitor PP2 alone did not influence the interaction of Src with either EGFR or caveolin-1. However, compared with TRAIL alone, a combination of PP 2 and TRAIL partially reversed the interaction of Src with both EGFR and caveolin-1. As before, the Src-EGFR and Src-caveolin-1 interactions were also detected by Duolink (Fig. 5B), and PP2 was shown to partially inhibit these TRAIL-induced interactions (Fig. 5B). However, knockdown of caveolin-1 did not influence the interaction of Src and EGFR (Fig. 5C). These results indicate that the TRAIL-induced Src activation triggered the interaction of Src with both EGFR and caveolin-1. 

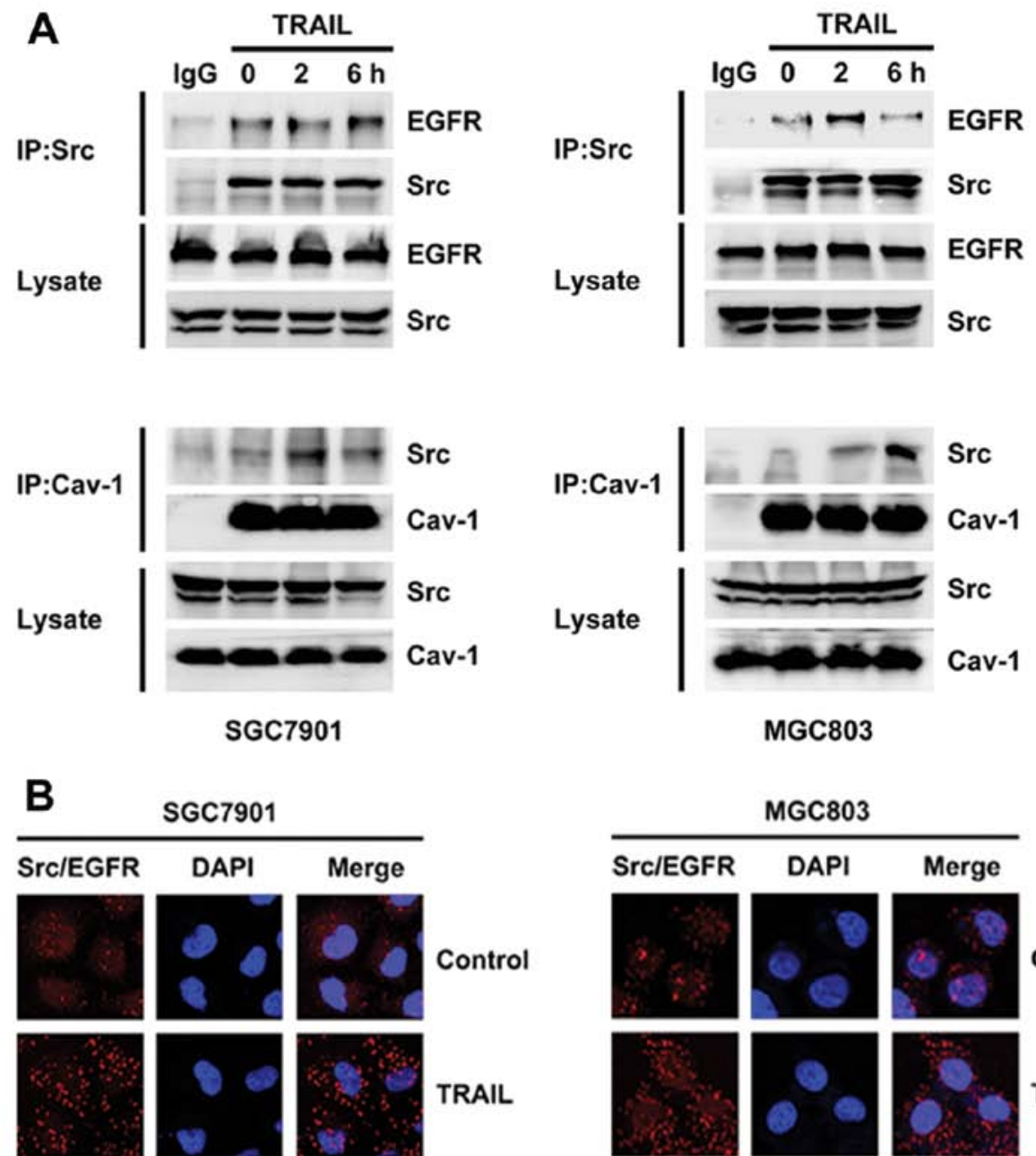

SGC7901

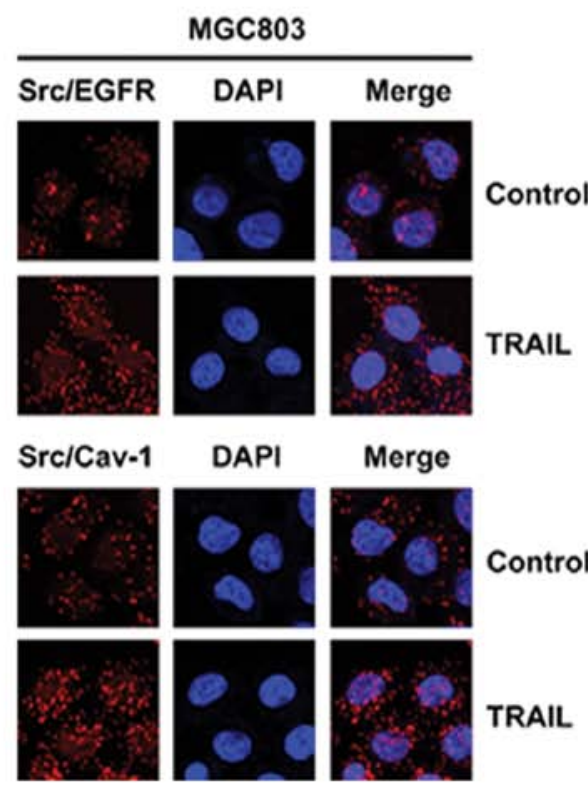

Figure 4. TRAIL induces the interaction of Src with both EGFR and caveolin-1 in gastric cancer cells. (A) SGC-7901 and MGC-803 cells were treated with $100 \mathrm{ng} / \mathrm{ml}$ TRAIL for 2 and $6 \mathrm{~h}$, and then the interaction of Src with EGFR and caveolin-1 was detected by immunoprecipitation. (B) SGC-7901 and MGC-803 cells were treated with $100 \mathrm{ng} / \mathrm{ml}$ TRAIL for $6 \mathrm{~h}$. The interaction of Src with EGFR and caveolin-1 was detected by Duolink in situ PLA. Red, the interaction of Src with EGFR or caveolin-1; blue, nuclear (magnification x60). TRAIL, tumor necrosis factor-related apoptosis-inducing ligand; EGFR, epidermal growth factor receptor.

\section{Discussion}

Caveolin-1-enriched lipid rafts provide an important platform for triggering receptor tyrosine kinase activation (15). For example, stretch-induced EGFR transactivation in mesangial cells requires caveolin-1 phosphorylation (9), while caveolin-1 also drives the internalization and nuclear transport of EGFR in the regulation of DNA repair in irradiated cells (16). However, it has not been determined whether caveolin-1 regulates TRAIL-induced EGFR activation. In the present study, TRAIL was shown to induce EGFR translocation into lipid rafts, leading to the activation of EGFR and caveolin-1 in gastric cancer SGC-7901 and MGC-803 cells. Knockdown of caveolin-1 partially inhibited EGFR activation and enhanced TRAIL sensitivity, indicating that caveolin-1 promotes TRAIL-induced EGFR activation and antagonizes TRAILtriggered apoptosis.

EGFR activation is also regulated by the non-receptor tyrosine kinase Src, and the Src family kinase regulates caveolin-1 activation. Src activation was shown to be essential for ATP-dependent activation of the sheddase ADAM17, which is responsible for EGFR activation in airway epithelial cells (17), while a blockade of Src signaling significantly increased TRAIL-induced cell death in lapatinib (a dual EGFR and ErbB-2 tyrosine kinase inhibitor)-resistant breast cancer cells (18). It was also reported that Src-dependent cave- 
A

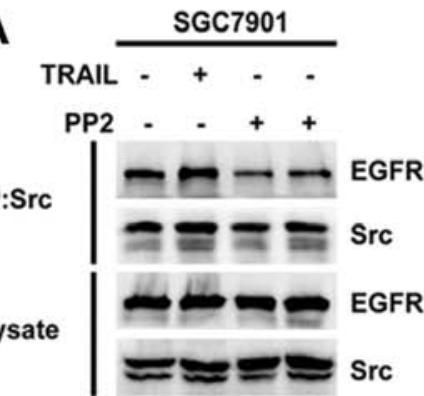

A

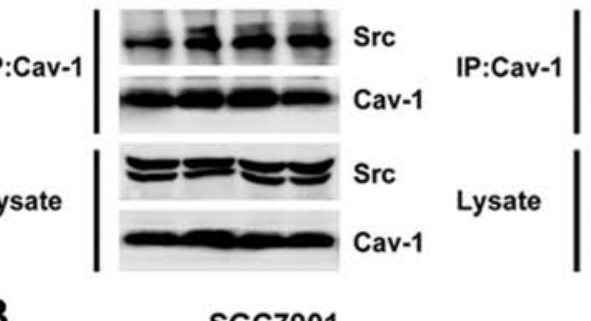

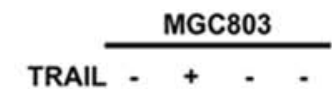

IP:Src

PP2 - - + +

Lysate $\mid=-2 \mathrm{SGF}$

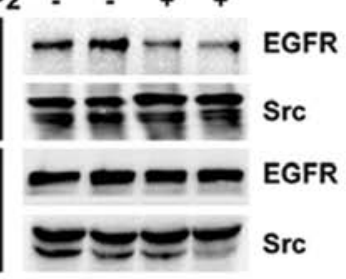

C siRnA $\frac{\text { NS }}{\frac{\text { SGC7901 }}{-+} \frac{\text { Cav-1 }}{-+}}$
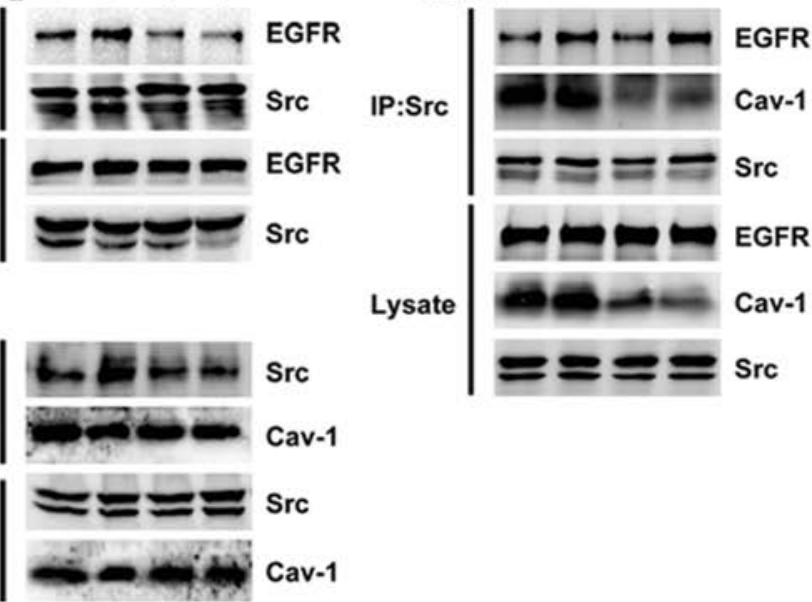

B

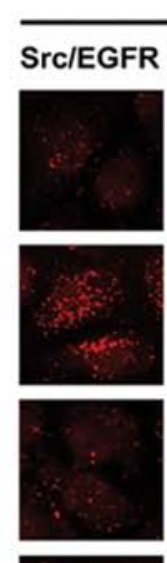

SGC7901

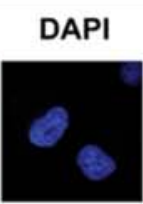

Merge

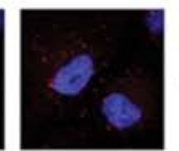

Control
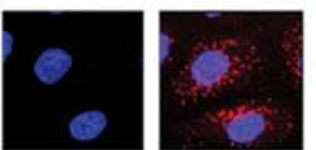

TRAIL
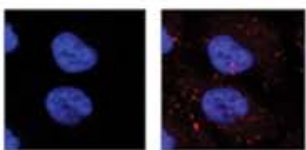

PP2
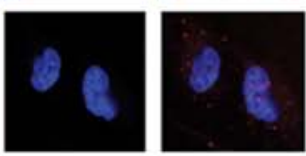

PP2, TRAIL

SGC7901

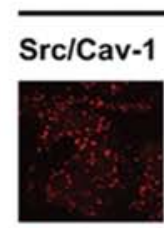

DAPI
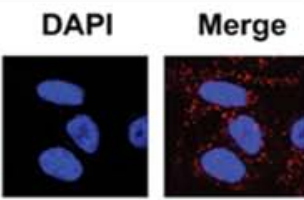

Control
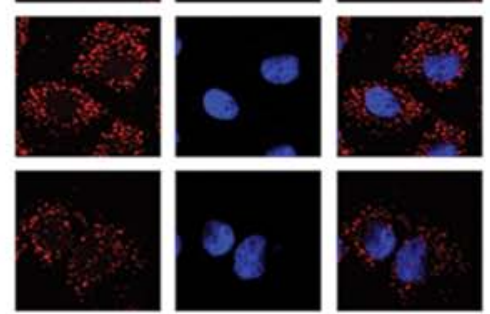

TRAIL

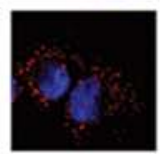

PP2
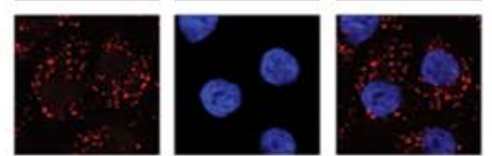

PP2, TRAIL
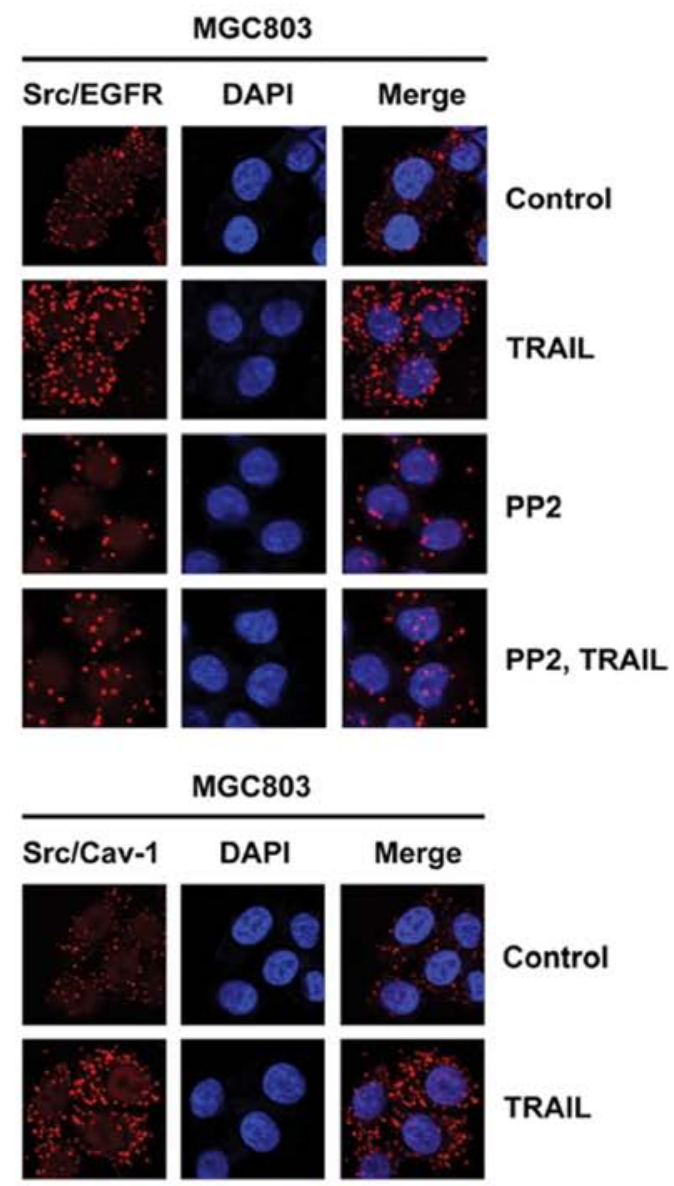

TRAIL
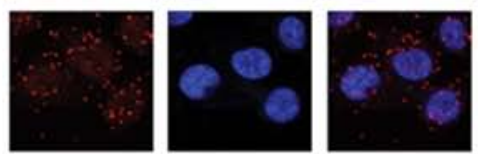

PP2
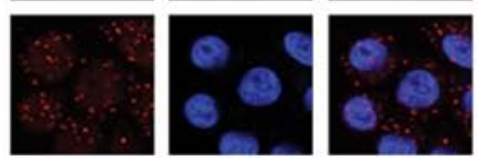

PP2, TRAIL

Figure 5. Inhibition of Src activation prevents the interaction of Src with both EGFR and caveolin-1 in gastric cancer cells. (A) SGC-7901 and MGC-803 cells were preincubated with $10 \mu \mathrm{M}$ Src inhibitor PP 2 for $1 \mathrm{~h}$, and then treated with $100 \mathrm{ng} / \mathrm{ml}$ TRAIL for $6 \mathrm{~h}$. The interaction of Src with EGFR and caveolin-1 was detected by immunoprecipitation. (B) SGC-7901 and MGC-803 cells were treated under similar conditions to those in A. The interaction of Src with EGFR and caveolin-1 was detected by Duolink in situ PLA. Red, the interaction of Src with EGFR or caveolin-1; blue, nuclear (magnification x60). (C) SGC-7901 cells were transiently transfected with caveolin-1 siRNA for $48 \mathrm{~h}$, followed by $100 \mathrm{ng} / \mathrm{ml}$ TRAIL for $6 \mathrm{~h}$. The interaction of Src with EGFR and caveolin-1 was detected by immunoprecipitation. EGFR, epidermal growth factor receptor; TRAIL, tumor necrosis factor-related apoptosis-inducing ligand. 
olin-1 phosphorylation was a critical event in transforming growth factor (TGF)- $\beta 1$-initiated SMAD2/3 activation (19), and Src-mediated shedding of TGF- $\alpha$ was found to be critical for TRAIL-induced EGFR activation in colorectal cancer cells (6). In the present study, TRAIL activated Src and induced its translocation into lipid rafts. PP2 inhibition of Src activation reduced the activation of caveolin-1 and EGFR, and increased TRAIL sensitivity. However, knockdown of caveolin-1 did not influence Src activation. Thus, it is conceivable that Src promotes caveolin-1-regulated EGFR activation and antagonizes TRAIL-induced gastric cancer cell apoptosis.

To identify the regulatory mechanism of Src and caveolin-1 involved in EGFR activation, the interaction of these molecules was investigated. Irradiation was shown to promote the stability of Src in complex with phosphorylated EGFR in bronchial carcinoma cells (20), while the phosphorylation of caveolin-1 by Src and subsequent binding of the Src SH2 domain to caveolin-1 led to the accumulation of activated Src in focal adhesions of COS-7 and REF52 cells (21). Moreover, in the angiotensin II-induced epithelial-to-mesenchymal transition, angiotensin II caused EGFR to associate with phosphorylated caveolin-1 and increased its phosphorylation in the caveolin-1-enriched fraction (22). However, the role of Src and caveolin-1 in the regulation of TRAIL-induced EGFR activation is not clear. In the present study, TRAIL was found to induce the interaction of Src with both EGFR and caveolin-1, but no interaction between EGFR and caveolin-1 was detected. The inhibition of Src activation partially reversed the interaction of Src with both EGFR and caveolin-1, while, conversely, knockdown of caveolin-1 had no effect on the Src-EGFR interaction. Thus, TRAIL induced EGFR activation at least partially by the interaction of both Src-EGFR and Src-caveolin-1.

In summary, we showed that Src activated EGFR through the interaction of both Src-EGFR and Src-caveolin-1, and then antagonized TRAIL-induced apoptosis in gastric cancer cells. It can therefore be hypothesized that the Src-caveolin-1-EGFR activation pathway is involved in the TRAIL resistance mechanism of certain cell types.

\section{Acknowledgements}

This study was supported by Chinese National Foundation of National Sciences grants (nos. 81201802, 81172369, 81172198,81372546 and 81372485), the Specialized Research Fund for the Doctoral Program of Higher Education (nos. 20102104120008 and 20112104110005), the National Science and Technology Major Project (no. 2013ZX09303002) and the Science and Technology Plan Project of Liaoning Province (no. 2011404013-1). The authors thank Jian Gao and Lu Yao (Experiment Technology Center of China Medical University) for kindly providing technical support.

\section{References}

1. Cervantes A, Roda D, Tarazona N, Roselló S and Pérez-Fidalgo JA: Current questions for the treatment of advanced gastric cancer. Cancer Treat Rev 39: 60-67, 2013.

2. Schaefer U, Voloshanenko O, Willen D and Walczak H: TRAIL: a multifunctional cytokine. Front Biosci 12: 3813-3824, 2007.
3. Morizot A, Mérino D, Lalaoui N, et al: Chemotherapy overcomes TRAIL-R4-mediated TRAIL resistance at the DISC level. Cell Death Differ 18: 700-711, 2011.

4. Xu L, Qu X, Luo Y, et al: Epirubicin enhances TRAIL-induced apoptosis in gastric cancer cells by promoting death receptor clustering in lipid rafts. Mol Med Rep 4: 407-411, 2011.

5. Cho IR, Koh SS, Min HJ, et al: Reovirus infection induces apoptosis of TRAIL-resistant gastric cancer cells by downregulation of Akt activation. Int J Oncol 36: 1023-1030, 2010.

6. Van Schaeybroeck S, Kelly DM, Kyula J, Stokesberry S, Fennell DA, Johnston PG and Longley DB: Src and ADAM-17mediated shedding of transforming growth factor- $\alpha$ is a mechanism of acute resistance to TRAIL. Cancer Res 68: 8312-8321, 2008

7. Xu L, Zhang Y, Liu J, et al: TRAIL-activated EGFR by Cbl-b-regulated EGFR redistribution in lipid rafts antagonises TRAIL-induced apoptosis in gastric cancer cells. Eur J Cancer 48: 3288-3299, 2012.

8. Wang S, Yu S, Shi W, Ge L, Yu X, Fan J and Zhang J: Curcumin inhibits the migration and invasion of mouse hepatoma Hca-F cells through down-regulating caveolin-1 expression and epidermal growth factor receptor signaling. IUBMB Life 63: 775-782, 2011.

9. Zhang B, Peng F, Wu D, Ingram AJ, Gao B and Krepinsky JC: Caveolin-1 phosphorylation is required for stretch-induced EGFR and Akt activation in mesangial cells. Cell Signal 19: 1690-1700, 2007.

10. Zhang J, Kalyankrishna S, Wislez M, et al: SRC-family kinases are activated in non-small cell lung cancer and promote the survival of epidermal growth factor receptor-dependent cell lines. Am J Pathol 170: 366-376, 2007.

11. Wu T, Zhang B, Ye F and Xiao Z: A potential role for caveolin-1 in VEGF-induced fibronectin upregulation in mesangial cells: involvement of VEGFR2 and Src. Am J Physiol Renal Physiol 304: F820-F830, 2013.

12. Chen Z, Bakhshi FR, Shajahan AN, et al: Nitric oxide-dependent Src activation and resultant caveolin-1 phosphorylation promote eNOS/caveolin-1 binding and eNOS inhibition. Mol Biol Cell 23: 1388-1398, 2012

13. Wang $\mathrm{Y}$, Roche $\mathrm{O}, \mathrm{Xu} \mathrm{C}$, et al: Hypoxia promotes ligandindependent EGF receptor signaling via hypoxia-inducible factor-mediated upregulation of caveolin-1. Proc Natl Acad Sci USA 109: 4892-4897, 2012.

14. Xu L, Qu X,Zhang Y, et al: Oxaliplatin enhances TRAIL-induced apoptosis in gastric cancer cells by CBL-regulated death receptor redistribution in lipid rafts. FEBS Lett 583: 943-948, 2009.

15. Irwin ME, Mueller KL, Bohin N, Ge Y and Boerner JL: Lipid raft localization of EGFR alters the response of cancer cells to the EGFR tyrosine kinase inhibitor gefitinib. J Cell Physiol 226: 2316-2328, 2011

16. Dittmann K, Mayer C, Kehlbach R and Rodemann HP: Radiation-induced caveolin-1 associated EGFR internalization is linked with nuclear EGFR transport and activation of DNA-PK. Mol Cancer 7: 69, 2008.

17. Sham D, Wesley UV, Hristova $M$ and van der Vliet A: ATP-mediated transactivation of the epidermal growth factor receptor in airway epithelial cells involves DUOX1-dependent oxidation of Src and ADAM17. PLoS One 8: e54391, 2013.

18. De Luca A, D'Alessio A, Gallo M, Maiello MR, Bode AM and Normanno N: Src and CXCR4 are involved in the invasiveness of breast cancer cells with acquired resistance to lapatinib. Cell Cycle 13: 148-156, 2014.

19. Samarakoon R, Chitnis SS, Higgins SP, Higgins CE, Krepinsky JC and Higgins PJ: Redox-induced $S r c$ kinase and caveolin-1 signaling in TGF- $\beta 1$-initiated SMAD2/3 activation and PAI-1 expression. PLoS One 6: e22896, 2011.

20. Dittmann K, Mayer C, Kehlbach R, Rothmund MC and Peter Rodemann H: Radiation-induced lipid peroxidation activates src kinase and triggers nuclear EGFR transport. Radiother Oncol 92: 379-382, 2009.

21. Gottlieb-Abraham E, Shvartsman DE, Donaldson JC, Ehrlich M, Gutman O, Martin GS and Henis YI: Src-mediated caveolin-1 phosphorylation affects the targeting of active Src to specific membrane sites. Mol Biol Cell 24: 3881-3895, 2013.

22. Chen J, Chen JK and Harris RC: Angiotensin II induces epithelialto-mesenchymal transition in renal epithelial cells through reactive oxygen species/Src/caveolin-mediated activation of an epidermal growth factor receptor-extracellular signal-regulated kinase signaling pathway. Mol Cell Biol 32: 981-991, 2012. 